\title{
Disociaciones entre prosodia emocional y lingüística en pacientes con lesiones cerebrales del hemisferio derecho
}

\author{
Dissociations between emotional and linguistic prosody in patients with right hemisphere \\ lesions
}

\author{
Samanta Leivaa ${ }^{\mathrm{a}, *}$, Micaela Difalcis ${ }^{\mathrm{a}, \mathrm{c}}$, Cynthia López ${ }^{\mathrm{a}}$, Laura Margulis ${ }^{\mathrm{a}, \mathrm{d}}$, Andrea Micciullid, \\ Valeria Abusamra ${ }^{\mathrm{a}, \mathrm{b}, \mathrm{c}}$, Aldo Ferreres ${ }^{\mathrm{a}, \mathrm{d}}$

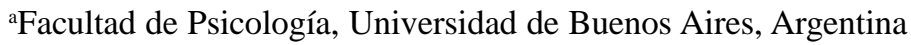 \\ ${ }^{b}$ Facultad de Filosofía y Letras, Universidad de Buenos Aires, Argentina \\ 'Consejo Nacional de Investigaciones Científicas y Técnicas (CONICET), Argentina \\ ¿Unidad de Neuropsicología, Hospital Interzonal General de Agudos «Eva Perón», Argentina
}

\section{Resumen}

El objetivo de este trabajo fue evaluar patrones de disociación entre el procesamiento de prosodia lingüística y emocional con el fin de analizar la independencia funcional entre ambos. Se realizó un estudio con un diseño de múltiples casos únicos de 17 pacientes con lesiones del hemisferio derecho debidas a accidente cerebro vascular. Se analizaron los perfiles de alteración/ conservación de la capacidad para procesar ambos tipos de prosodia con cuatro pruebas de comprensión y repetición de prosodia emocional y lingüística, y se comparó el rendimiento de cada paciente con un grupo control sano. Los resultados mostraron rendimiento disociado entre prosodia emocional y lingüística en la habilidad de comprensión prosódica en 3/17 pacientes y no se hallaron disociaciones dobles. El hallazgo de distintos patrones de alteración/conservación entre prosodia lingüística y emocional, tanto en la habilidad para comprender como para repetir prosodia, es evidencia que apoya el supuesto de independencia funcional entre los procesamientos de ambos tipos de prosodia.

Palabras clave: neuropsicología, prosodia, emoción, lenguaje, hemisferio derecho.

Para citar este artículo:

Leiva, S., Difalcis, M., López, C., Margulis, L., Micciulli, A., Abusamra, V., \& Ferreres, A. (2017). Disociaciones entre prosodia emocional y lingüística en pacientes con lesiones cerebrales del hemisferio derecho. Liberabit, 23(2), 211-232. doi: 10.24265/ liberabit.2017.v23n2.04

\section{Abstract}

The goal of this study was to evaluate dissociation patterns between processing of linguistic prosody and emotional (or affective) prosody, in order to analyze the functional independence between them. We carried out a multiple single-cases study of 17 patients with right hemisphere lesions due to stroke. The alteration / conservation profiles of the ability to process both types of prosody were analyzed with four tasks of comprehension and repetition of emotional and linguistic prosody. The performance of each patient was compared with a healthy control group. The results showed a dissociated performance between emotional and linguistic prosody in prosodic comprehension in 3/17 patients, and no double dissociations were found. Finding different patterns of alteration / conservation between linguistic and emotional prosody, both in the ability to understand and to repeat prosody, supports the assumption of functional independence between processing of both types of prosody.

Keywords: neuropsychology, prosody, emotion, language, right hemisphere.

Este es un artículo Open Access bajo la licencia Creative Commons Atribución-NoComercial-CompartirIgual 4.0

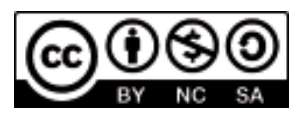




\section{Introducción}

Un mensaje verbal no contiene únicamente información sobre lo que se dice sino que, además, incluye claves o pistas sobre cómo se dice. La prosodia es uno de los componentes suprasegmentales del habla que es utilizado para comprender o expresar intenciones comunicativas a través de la melodía de la voz e incluye las variaciones del tono, el ritmo y la acentuación de los enunciados vocales (Ross \& Mesulam, 1979). La propuesta de Monrad (1947) fue una de las primeras clasificaciones en diferenciar distintos tipos de prosodia y, hasta la actualidad, la mayoría de los investigadores asumen que existen al menos dos tipos diferentes: la prosodia emocional y la lingüística. La prosodia emocional es aquella que se genera cuando el hablante expresa contenidos afectivos en el mensaje tales como alegría, miedo o enojo e incluye tres rasgos prosódicos: longitud, intensidad y tono de los sonidos del habla (Snow, 2000). Por otro lado, la prosodia lingüística es utilizada para segmentar las frases habladas al cumplir el rol equivalente a la puntuación para los textos escritos e incluye tres fenómenos: acento léxico, acento enfático y modalidad (Joanette et al., 2008). El acento léxico es aquel que permite diferenciar palabras que tienen igual composición fonémica. En español, por ejemplo, permite la diferenciación entre las palabras ‘salto' y 'saltó'. El acento enfático permite hacer hincapié o resaltar una parte del enunciado verbal por sobre otro. Por ejemplo, en la frase 'María salió de viaje' un énfasis sobre la palabra 'María' permite resaltar que fue María quien salió de viaje y no otra persona. Por último, la modalidad permite transmitir claves sobre la intencionalidad del hablante a través de la pronunciación de distintas formas tonales a nivel oracional. Por ejemplo, el enunciado 'Juan saluda al vecino’ se puede pronunciar en forma afirmativa, interrogativa o como una orden.

A pesar de que esta diferenciación dicotómica entre prosodia emocional y lingüística se encuentra aceptada en la actualidad, sigue siendo tema de debate si ambos tipos de prosodia son funcionalmente independientes. Por ejemplo, Snow (2000) ha planteado que no existe suficiente evidencia para afirmar una independencia total entre prosodia emocional y lingüística. Según este autor, ambos tipos de prosodia son en sí mismos lingüísticos y emocionales desde un punto de vista funcional, ya que las marcas tonales que permiten diferenciar, por ejemplo, que un sujeto está haciendo un pedido o una pregunta pueden ser interpretadas como formas actitudinales/emocionales que toma el hablante, en este caso, seguro de sí mismo o dubitativo. Asimismo, este autor postuló que los estados emocionales influyen en la forma en la que se modulan los tonos lingüísticos, ya sea elevando el tono si el sujeto se siente alegre, o bajándolo en el caso de tristeza. También se podría especular que el uso combinado es una opción del hablante que no descarta la independencia funcional.

Las evidencias a favor de la independencia entre prosodia emocional y lingüística han provenido fundamentalmente de dos grupos de estudios. Por un lado, de aquellos trabajos en los que se ha estudiado la implicancia diferencial o compartida de distintas partes del cerebro encargadas de procesar ambos tipos de prosodia, realizados con sujetos sanos e imágenes funcionales; $y$, por otro, de estudios con pacientes con lesiones cerebrales adquiridas con afectación disociada o no para procesar prosodia lingüística y emocional.

Respecto de las evidencias provenientes de estudios con imágenes funcionales, un reciente metaanálisis realizado sobre datos de 29 experimentos independientes en participantes sin patologías mostró que, si bien existen áreas cerebrales compartidas para el procesamiento de ambos tipos de prosodia, también hay estructuras cerebrales independientes para cada una (Belyk \& Brown, 2014). En esos estudios, la comprensión tanto de prosodia emocional como lingüística activaron áreas posteriores del surco temporal superior y el área motora suplementaria, mientras que algunas áreas del giro frontal inferior 
tuvieron activación selectiva para una u otra, siendo la región orbital (área de Brodmann 47) selectiva para la prosodia emocional y la región opercular (área de Brodmann 44) para la prosodia lingüística (Belyk \& Brown, 2014).

Las investigaciones realizadas con pacientes con lesiones cerebrales se han centrado principalmente en aquellos con lesiones del hemisferio derecho (HD) $y$ en el rol dominante de éste para el procesamiento de la prosodia, ya que parten de presunciones teóricas clásicas que sostienen que el HD sería dominante para procesar diversos aspectos pragmáticos, incluyendo la prosodia, mientras que no lo sería para los aspectos nucleares del lenguaje (fonología, morfología y sintaxis). En este grupo de pacientes, varios estudios han mostrado consistentemente alteraciones tanto en la capacidad para comprender como para producir prosodia emocional y lingüística (Cohen, Riccio, \& Flannery, 1994; Guranski \& Podemski, 2015; Harciarek, Heilman, \& Jodzio, 2006; Heilman, Leon, \& Rosenbek, 2004; Ross \& Monnot, 2008; Zaidel, Kasher, Soroker, \& Batori, 2002). Sin embargo, estos trabajos no se han focalizado en el estudio de la independencia entre prosodia emocional y lingüística sino en la dominancia del HD respecto del hemisferio izquierdo (HI) para procesarlas. Es por ello que Snow (2000) afirmó que las evidencias provenientes de pacientes con lesiones del HD no suelen ser concluyentes respecto de una distinción entre prosodia emocional y lingüística. Eso se puede deber, en parte, a los enfoques metodológicos utilizados que no han permitido, hasta la actualidad, obtener esos datos, ya sea porque realizan únicamente estudios de grupo y no analizan las disociaciones (e.g., Guranski \& Podemski, 2015), o porque incluyen en la evaluación de los pacientes un único tipo de prosodia y no el otro (e.g., Harciarek et al., 2006).

En este sentido, se torna de particular relevancia utilizar diseños y metodologías novedosos de estudios de caso único o múltiples casos únicos para aportar evidencia que compare directamente la disociación (o no) entre la prosodia lingüística y emocional en pacientes con lesiones del HD. Hallar patrones de disociaciones clásicas, dobles o fuertes al analizar patrones de alteración/conservación en los pacientes se convertiría en evidencia que permitirían tener datos sólidos para asumir que hay independencia funcional entre dos procesos (Fellows, 2013). Una disociación clásica es hallada cuando un paciente muestra rendimiento afectado en la tarea «A» pero su rendimiento en la tarea «B» es normal y, si además se halla a otro paciente con el rendimiento opuesto (normal en la tarea «A» pero alterado en la tarea «B»), este patrón se denomina disociación doble (Ellis \& Young, 1992). Las disociaciones dobles son las evidencias más sólidas que aporta la neuropsicología para asumir independencia funcional entre procesos cognitivos (Ellis \& Young, 1992). En este caso, lo sería entre la capacidad de producir y comprender prosodia emocional y lingüística. Por otra parte, en las disociaciones denominadas fuertes, se observa un patrón de alteración en ambas tareas (tomando el ejemplo anterior, tanto la tarea A como la B con rendimiento disminuido) pero el paciente tiene un rendimiento significativamente más afectado en una que otra (Shallice, 1988). La base para decir que el rendimiento está disociado a pesar de que esté afectado en ambas tareas es que el paciente tiene una discrepancia anormalmente grande entre las dos tareas, discrepancia que no se observa en los sujetos control.

En los últimos años, los estudios de casos únicos son cada vez más rigurosos y utilizan diseños de casocontrol y análisis estadísticos específicos que permiten demostrar de manera precisa la disociación (Crawford \& Garthwaite, 2012; McIntosh \& Brooks, 2011), pero estos métodos nunca han sido utilizados para analizar disociaciones entre la prosodia emocional y lingüística en los pacientes con lesiones focales del HD.

El objetivo de este trabajo, entonces, fue llevar a cabo un estudio de múltiples casos únicos para analizar la presencia de diferentes patrones de 
disociaciones (clásicas, dobles y fuertes) entre el procesamiento de prosodia emocional y lingüística en pacientes con lesiones del HD. La relevancia y novedad de este trabajo reside en que, como se describió anteriormente, aunque se han reportado alteraciones frecuentes en el procesamiento prosódico en general de estos pacientes, no se ha estudiado en la literatura la independencia funcional con las metodologías mencionadas que son las que permitirían aportar datos fundamentales para asumir dicha independencia entre los dos tipos de prosodia.

\section{Metodología}

\section{Participantes}

La inclusión del grupo de pacientes se realizó en el marco de un proyecto de investigación UBACyT desarrollado durante los años 2011-2014, de la Facultad de Psicología de la Universidad de Buenos Aires, que tuvo como objetivo evaluar las alteraciones de la comunicación verbal en pacientes con lesiones del HD. Para la presente investigación, se evaluaron 17 pacientes con lesiones únicas del HD debidas a accidentes cerebrovasculares (ACV), 6 mujeres y 11 hombres, con una edad media en años de 58 (10.4) y una escolaridad media en años de 9.7 (4.9). En la Tabla 1 se muestran los datos demográficos y neurológicos de cada paciente. Todos fueron reclutados y evaluados en la Unidad de Neuropsicología del Hospital Interzonal General de Agudos «Eva Perón» de San Martín, provincia de Buenos Aires, Argentina. Los pacientes fueron derivados por los médicos neurólogos a cargo de su tratamiento clínico, quienes les propusieron participar en esta investigación. Los criterios de inclusión/ exclusión fueron: 1) ACV isquémico o hemorrágico que afecte únicamente el HD con al menos tres meses de evolución, documentado por estudios de tomografía axial computarizada (TAC) o resonancia magnética nuclear (RMN); 2) que se encuentren con seguimiento ambulatorio al momento de la evaluación; 3) ausencia de alteraciones sensoriomotoras graves o heminegligencia; 4) sin otros antecedentes neurológicos y/o psiquiátricos adicionales; 5) sin patologías clínicas adicionales que puedan afectar la cognición (e.g., hipotiroidismo); 6) ausencia de alteraciones motoras y/o sensitivas que afecten la mano dominante; 7) ausencia de antecedentes de alteraciones de adquisición del lenguaje, lectura o escritura; 8) lengua nativa español. El cumplimiento de los criterios de inclusión/exclusión fue realizado por dos médicos neurólogos especialistas en neuropsicología o neuropsiquiatría, quienes evaluaron cada caso teniendo en cuenta la información de la historia clínica de los pacientes, datos recabados de una entrevista semi-estructurada y análisis de las imágenes cerebrales estructurales (TAC y/o RMN).

Con el fin de que cada paciente tenga un grupo control propio emparejado según la edad y la escolaridad, para obtener los datos de rendimiento normal en las pruebas, se formaron 17 grupos, uno para cada paciente, compuestos por 15 participantes adultos sanos cada uno. Toda la información de los participantes de este grupo, incluyendo la demográfica y los puntajes de rendimiento en las pruebas utilizadas (ver Materiales y Procedimiento) fue extraída de la base de datos normativos de la adaptación argentina del Protocolo para la Evaluación de la Comunicación de Montréal (Protocolo MEC, Ferreres et al., 2007). Dicha base fue obtenida a través del primer autor de la adaptación mencionada. Los criterios de inclusión/exclusión para los participantes de los grupos control que se utilizó en el estudio de Ferreres et al. (2007) fueron: 1) participantes sin alteraciones neurológicas 0 psiquiátricas; 2) sin antecedentes de abuso de alcohol o drogas; 3) que sepan leer y escribir; y 4) con lengua materna español, variedad del Río de la Plata. Para el presente trabajo se seleccionaron 15 participantes de la base de datos normativos de forma aleatoria para cada paciente, teniendo en cuenta el rango de edad y escolaridad de los mismos para que no haya diferencias significativas entre los datos demográficos del grupo control y cada paciente. No se emparejó a los grupos de acuerdo al género ya que los autores de la adaptación argentina no reportaron diferencias 


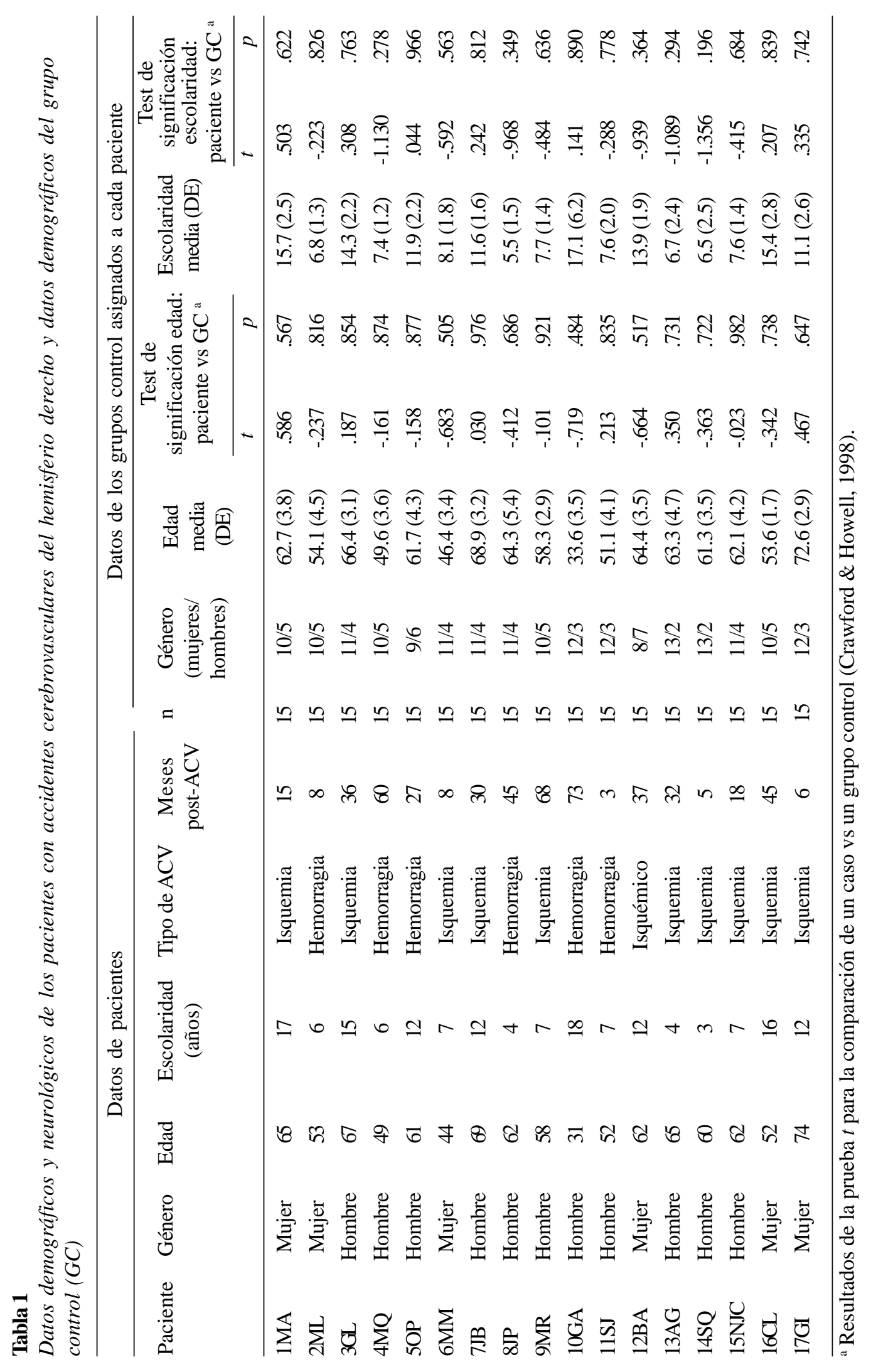


estadísticamente significativas entre hombres y mujeres. En la Tabla 1 se muestran los datos demográficos de cada uno de los grupos control asignados para cada paciente, así como los resultados de la comparación estadística de la edad y la escolaridad entre pacientes y controles.

\section{Materiales y procedimiento}

A todos los pacientes evaluados se les solicitó el consentimiento informado de participación voluntaria de acuerdo a la Declaración de Helsinki.

Se utilizaron cuatro pruebas estandarizadas de evaluación de la comprensión y producción de prosodia lingüística y emocional. Las mismas forman parte del Protocolo MEC (Joanette, Ska, \& Côté, 2004; adaptado por Ferreres et al., 2007) y fueron administradas siguiendo las instrucciones provistas en el manual de la adaptación argentina. Además de su versión original canadiense y la adaptación argentina, el protocolo MEC ha sido adaptado a múltiples culturas: la versión en portugués de Brasil (Fonseca et al., 2008b), en portugués europeo (de Souza et al., 2015) y dos versiones breves, una en francés y otra en portugués (Casarin et al., 2014; Ferré, Lamelin, Côté, Ska, \& Joanette, 2011). Por otra parte, esta batería ha mostrado tener adecuados indicadores de confiabilidad con valores de alfa de Cronbach entre .70 y .90 para todas las tareas, así como buena confiabilidad interevaluadores y adecuada validez clínica al mostrar buena sensibilidad a las alteraciones de la comunicación verbal en pacientes con lesiones cerebrales del hemisferio derecho (Becker, de Lima Müller, de Carvalho Rodrigues, Villavicencio, \& Fumagalli de Salles, 2014; de Lima Muller, Becker, \& Fumagalli de Salles, 2014; de Oliveira, Carlesso, de Feritas, Saloum, \& Fonseca, 2016; Ferré et al., 2009; Lajoie, Ferré, \& Ska, 2010; Fonseca et al., 2008a; Zimmermann, Gindri, de Oliveira, \& Fonseca, 2011).
A continuación se describe cada una de las tareas:

a) Prosodia Lingüística - Comprensión:

Evalúa la capacidad de reconocer auditivamente si la prosodia de una oración es afirmativa, interrogativa o imperativa. Se explica al participante que escuchará oraciones y que su tarea consiste en identificar si cada una de ellas se trata de una pregunta, una afirmación o una orden, basándose únicamente en la entonación de la voz. La prueba incluye 12 estímulos grabados con cuatro oraciones base de contenido neutro compuestas por la forma sujeto-verbo-sujeto (e.g., «Pedro saluda al vecino») que se pronuncian con tres entonaciones: interrogativa, afirmativa y exclamativa. Los estímulos fueron presentados en el orden aleatorio prefijado según la estandarización. Luego de escuchar cada oración, los participantes debían calificar la entonación mediante el señalamiento de una de tres imágenes impresas en una tarjeta, correspondientes a cada opción posible («?» para interrogación; «.» para afirmación; y «!» para las órdenes). Esta prueba, así como las tres que se describen a continuación, tuvieron dos estímulos de práctica para asegurarse la correcta comprensión de las instrucciones de la tarea. Ninguna de las pruebas administradas tuvieron límite de tiempo para dar las respuestas $\mathrm{y}$, en todos los casos, los participantes podían solicitar que se repita el estímulo una única vez. En esta y en las subsiguientes pruebas, se registraron las respuestas dadas por los participantes y se asignó un punto por cada respuesta correcta (puntuación máxima posible para cada tarea: 12).

b) Prosodia Lingüística - Repetición:

Evalúa la capacidad de producir entonaciones lingüísticas (afirmativa, interrogativa o imperativa) luego de haberlas escuchado. Se presentaron a los participantes los mismos estímulos de la prueba anterior y se les pidió que los repitan respetando 
la entonación escuchada. Tal y como está consignado en el manual de administración y puntuación del protocolo utilizado, las respuestas dadas por los participantes fueron grabadas por las evaluadoras para que la puntuación se realice a posteriori, al escuchar con detalle las producciones y, así, determinar si la pronunciación de la prosodia fue correcta o incorrecta.

c) Prosodia Emocional - Comprensión:

Evalúa la capacidad de reconocimiento de prosodia emocional correspondiente a tres entonaciones afectivas: tristeza, alegría y enojo. Se explicó a los participantes que iban a escuchar oraciones y que su tarea consistiría en identificar si la persona que las pronunciaba está contenta, triste o enojada, basándose únicamente en la entonación de la voz. La prueba incluyó 12 estímulos grabados con cuatro oraciones base de contenido neutro compuestas por la forma sujetoverbo-sujeto (e.g., «Martín lee el diario») que se pronuncian con tres entonaciones emocionales: alegre, triste o enojado. Luego de escuchar cada estímulo, los participantes debían señalar una de tres imágenes representativas de la entonación escuchada (íconos con dibujos de una cara alegre, triste o enojada).

d) Prosodia Emocional - Repetición:

Evalúa la capacidad para producir prosodia emocional (triste, alegre o enojada), luego de haberla escuchado. Se presentó a los participantes nuevamente los 12 estímulos utilizados para la prueba anterior y se les pidió que los repitan respetando la entonación escuchada. Tal y como se mencionó para la otra tarea de producción prosódica, las respuestas verbales dadas por los participantes fueron grabadas y se realizó la puntuación de las mismas en una instancia posterior, en la que se escuchó con detalle las producciones realizadas en cada caso.

\section{Análisis de datos}

Se calculó el total de aciertos de cada paciente en las cuatro pruebas, así como las medias y desviaciones estándar de cada grupo control. Para analizar las disociaciones entre las tareas, se utilizaron los criterios operacionalizados por Crawford y Garthwaite (2005) para disociación clásica, doble o fuerte. Según estos autores, un paciente tiene una disociación clásica entre dos tareas cuando se cumplen los siguientes criterios: 1) el paciente presenta un rendimiento significativamente menor que el grupo control $(p<.05)$ en la tarea A; 2$)$ el paciente no muestra diferencias de rendimiento con el grupo control $(p>.05)$ en la tarea B; y 3) la discrepancia observada entre las tareas A y B en el paciente debe ser estadísticamente diferente $(p<.05)$ a la discrepancia observada en el grupo control entre las mismas tareas. Los criterios para una disociación doble requieren que en un paciente con rendimiento afectado en la tarea A se cumplan los criterios para disociación clásica con la tarea B y que, en otro paciente con rendimiento afectado en la tarea $\mathrm{B}$, se cumplan los criterios de disociación clásica con la tarea A, es decir, dos pacientes con patrones de disociación clásica opuestos. Finalmente, los criterios para que una disociación sea considerada fuerte establecen que un paciente que cumple el criterio 1 de disociación clásica para la tarea A, también presente un rendimiento significativamente menor $(p$ $<.05)$ que el grupo control en la tarea B (criterio 2) y que cumpla el criterio 3 mencionado para disociación clásica.

Para verificar cada uno de los criterios, se utilizaron los estadísticos sugeridos por los autores. El cumplimiento de los criterios 1 y 2 (para cualquier tipo de disociación) se testeó con la prueba $t$ modificada de una cola diseñada especialmente para casos únicos (Crawford \& Howell, 1998) que compara el rendimiento de un paciente con un grupo control compuesto por una muestra pequeña $(n<$ 50). Para el cumplimiento del tercer criterio, se utilizó el Revised Standarized Difference Test (RSDT), 
que compara la discrepancia entre las tareas en el paciente y en el grupo control teniendo en cuenta la correlación entre las mismas (Crawford \& Garthwaite, 2005). Además, se realizó una estimación del porcentaje de la población que presentaría un rendimiento más bajo que los pacientes para cada una de las pruebas (Crawford \& Garthwaite, 2002). Por último, se reporta el tamaño del efecto $\left(Z_{c c}\right)$ de todas las comparaciones, de acuerdo con lo propuesto por Crawford, Garthwaite y Porter (2010). Los análisis se realizaron con los programas Dissocs_ES y Singlims_ES. Ambos pueden ser descargados gratuitamente de la página web del autor citado. Los análisis se realizaron para estudiar la presencia de disociaciones entre las siguientes pruebas:

- Prosodia Lingüística - Comprensión vs Prosodia Emocional - Comprensión.

- Prosodia Lingüística - Repetición vs Prosodia Emocional - Repetición.

\section{Resultados}

En la tabla 2 se muestran las puntuaciones obtenidas por cada uno de los pacientes en las cuatro pruebas administradas, las medias y $D E$ de acierto de cada grupo control así como los resultados de la comparación estadística entre pacientes y controles. Se observó que 5/17 pacientes (casos 1, 2, 7, 12 y 14) presentaron un rendimiento significativamente menor que el grupo control en la prueba Prosodia Lingüística - Comprensión, 4/17 pacientes (casos 1, 4, 14 y 15) en Prosodia Lingüística - Repetición, 6/ 17 pacientes (casos 1, 2, 3, 5, 7 y 12) en Prosodia Emocional - Comprensión y 8/17 (casos 3, 5, 6, 7, 9, 11, 12 y 14) en Prosodia Emocional - Repetición. Cinco pacientes (casos 8, 10, 13, 16 y 17) no tuvieron diferencias estadísticamente significativamente con el grupo control en ninguna de las cuatro pruebas administradas. 


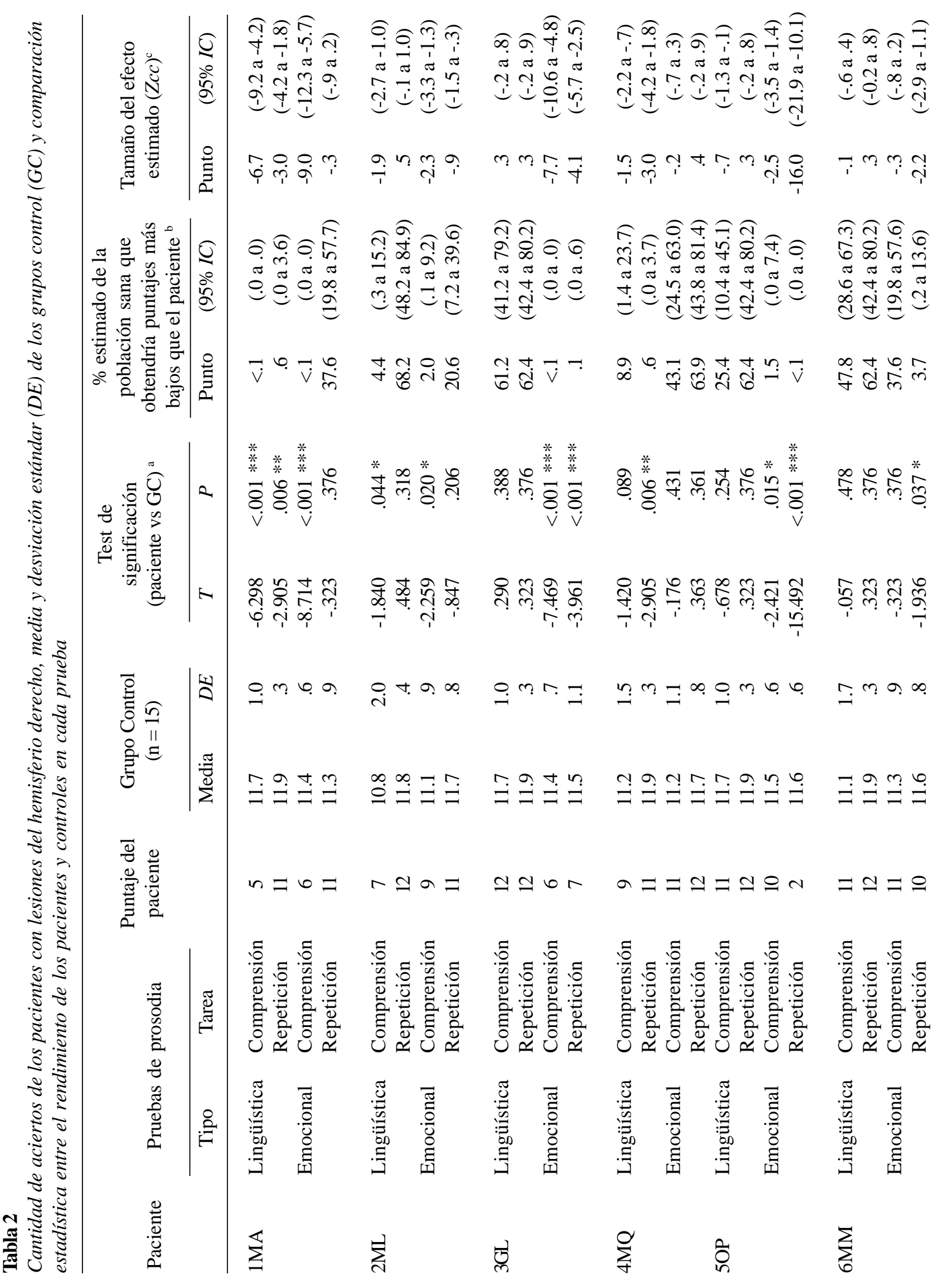



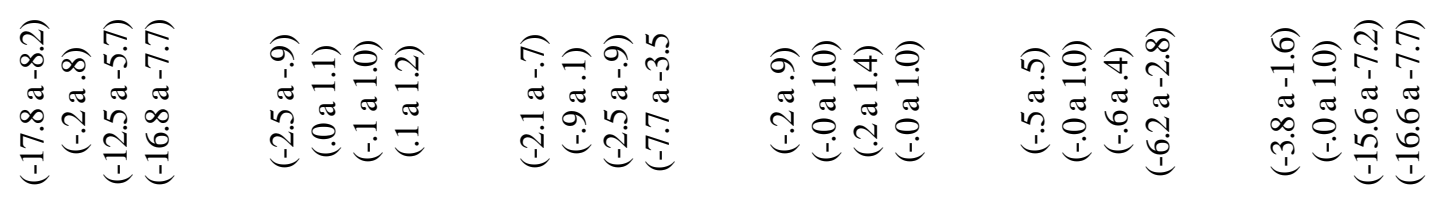

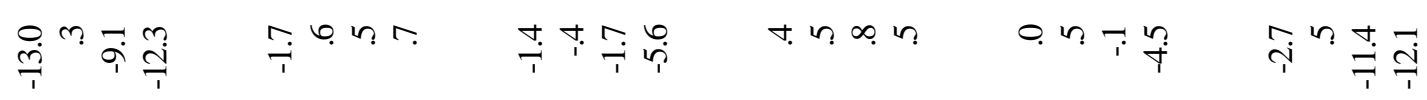

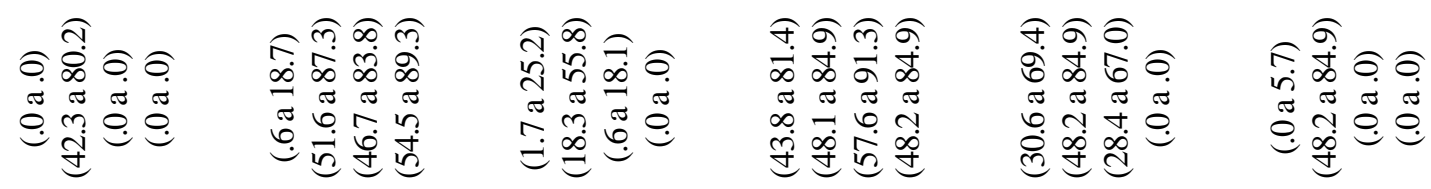

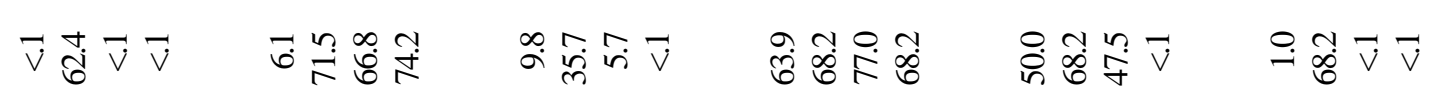

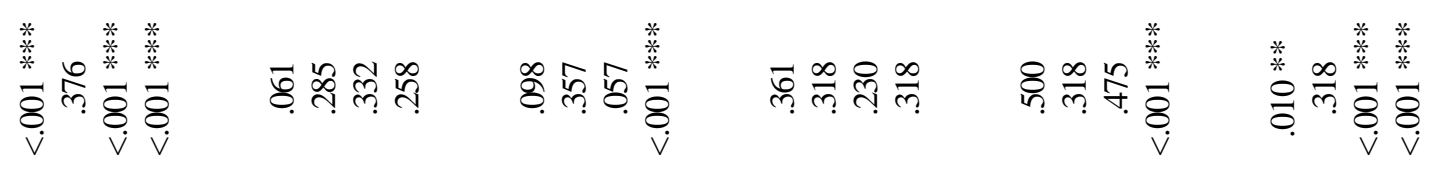

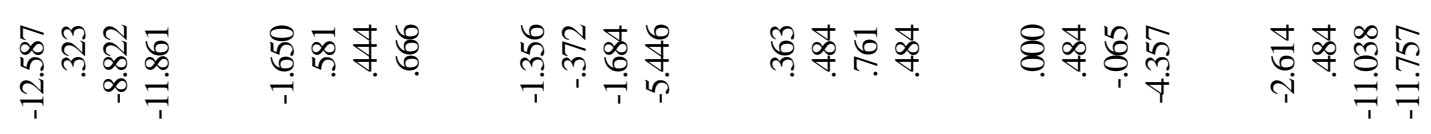

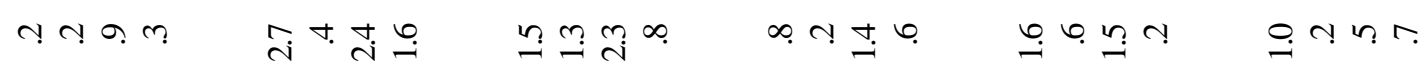

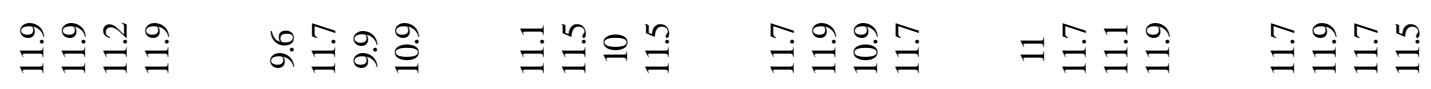

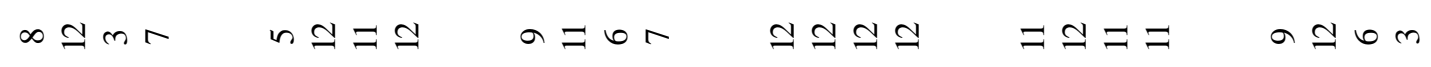

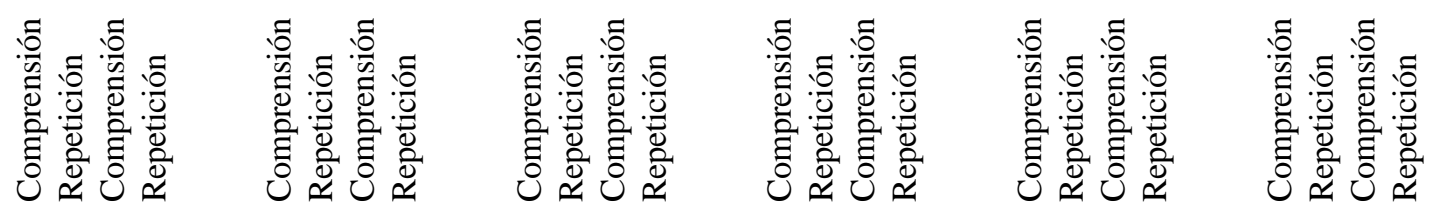

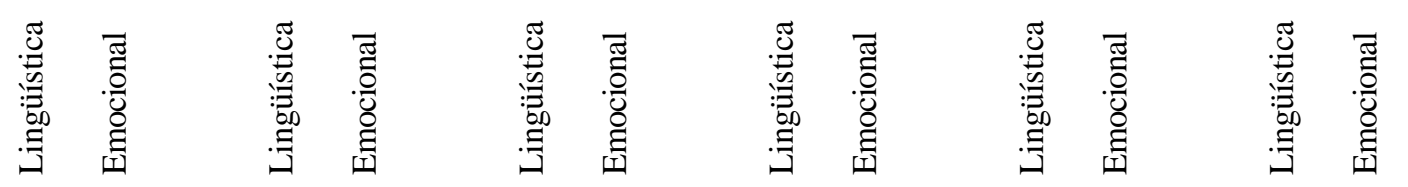

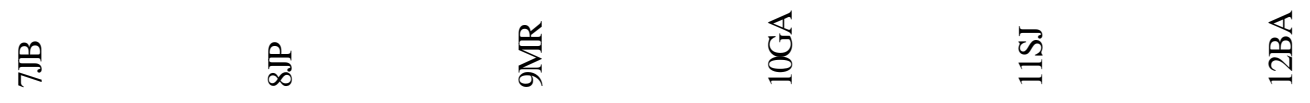




\begin{tabular}{|c|c|c|c|c|c|}
\hline 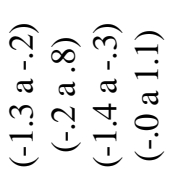 & 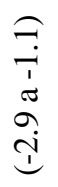 & 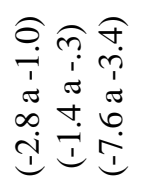 & 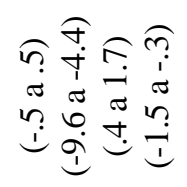 & 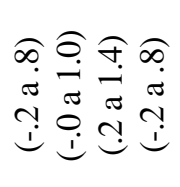 & 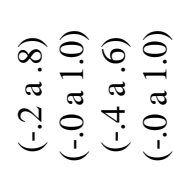 \\
\hline ด & $\stackrel{\circ}{i}$ & 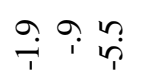 & 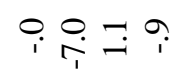 & n & 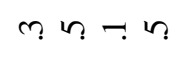 \\
\hline 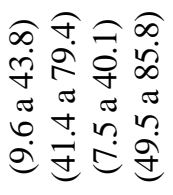 & 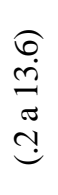 & 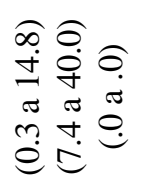 & 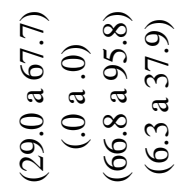 & 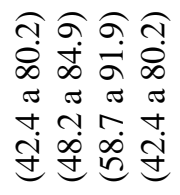 & 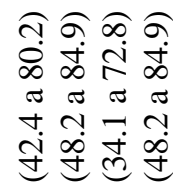 \\
\hline 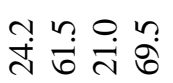 & $\hat{m}$ & 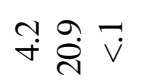 & 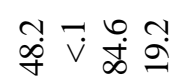 & 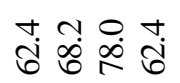 & 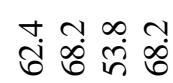 \\
\hline
\end{tabular}

ปุ

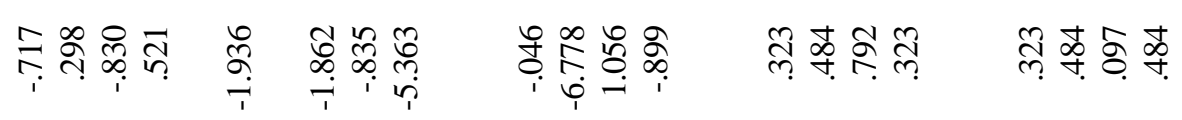

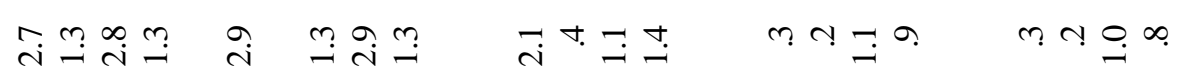

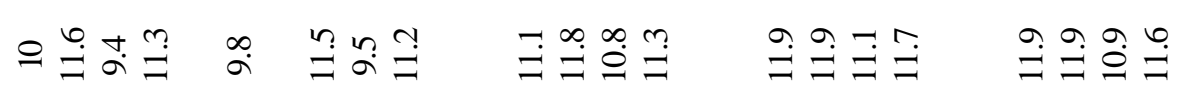

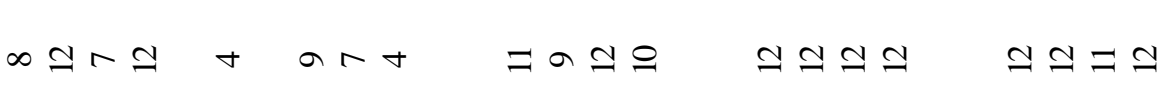

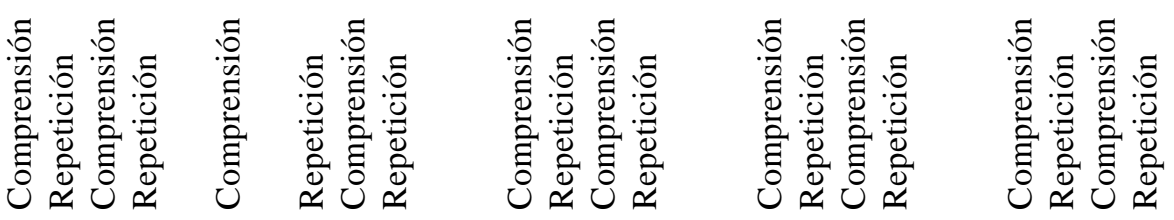

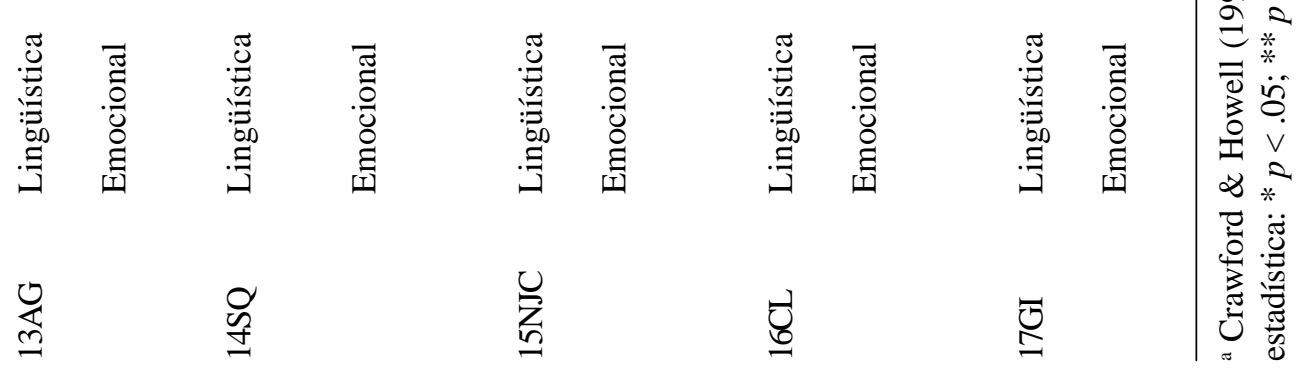




\section{Disociaciones en la capacidad de comprensión prosódica.}

Como se muestra en la Tabla 2, los pacientes 4, $6,8,9,10,11,13,15,16$ y 17 tuvieron rendimiento sin diferencias significativas con los controles en ambas pruebas de comprensión prosódica (lingüística y emocional), por lo que en ellos no se cumple con el criterio 1 para ningún tipo de disociación (clásica o fuerte).

Siete pacientes (casos 1, 2, 3, 5, 7, 12 y 14) tuvieron rendimiento estadísticamente menor que los controles en al menos una prueba de comprensión prosódica, cumpliendo con el criterio 1 para disociación clásica o fuerte. En la Tabla 3 se muestra la clasificación del rendimiento de los pacientes en ambas pruebas de comprensión prosódica (emocional y lingüística). Como se observa en dicha tabla, tres de los siete pacientes mencionados (casos 3,5 y 14) cumplieron además con el criterio 2 para disociación clásica ya que no tuvieron diferencias significativas con los controles en la otra prueba. Los pacientes 1 , 2, 7 y 12 rindieron significativamente peor que los controles tanto en la prueba de comprensión de prosodia emocional como en la de prosodia lingüística, cumpliendo así con los criterios 1 y 2 para disociación fuerte.

En la Tabla 3 se muestran, además, los resultados de la prueba RSDT. La misma fue utilizada para analizar si hubo diferencias significativas entre la discrepancia observada entre las tareas de comprensión en el paciente y la discrepancia observada en el grupo control entre las mismas tareas (criterio 3 para disociación clásica o fuerte). Los resultados del RSDT para las pruebas de comprensión prosódica mostraron que sólo el paciente 3 cumplió el tercer criterio para disociación clásica. Por su parte, los pacientes 7 y 12 lo hicieron para disociación fuerte. En los pacientes 1, 2, 5 y 14 no se observó disociaciones entre la comprensión de prosodia lingüística y emocional, ya que la discrepancia entre las pruebas de los mismos no tuvo diferencias estadísticamente significativas respecto de la discrepancia observada en el grupo control (criterio 3).

\section{Disociaciones en la capacidad de repetición prosódica.}

Como se muestra en la Tabla 2, los pacientes 2, 8, 10, 13, 16 y 17 tuvieron rendimiento sin diferencias estadísticamente significativas respecto de los controles en ambas pruebas de repetición prosódica. En ellos no se cumplió con el criterio 1 para ningún tipo de disociación. El resto de los pacientes tuvo rendimiento estadísticamente menor que los controles en al menos una de esas pruebas, cumpliendo con el criterio 1 para disociación clásica o fuerte. Diez de ellos cumplieron, además, con el criterio 2 para disociación clásica ya que no tuvieron rendimiento estadísticamente menor que los controles en la otra prueba. Solo un paciente (caso 14) cumplió con el segundo criterio para disociación fuerte, al tener rendimiento afectado en las pruebas de repetición con ambos tipos de prosodia.

En la Tabla 3 se muestran los resultados de la prueba RSDT. Estos mostraron que los pacientes 3, $5,7,9,11,12$ y 15 cumplieron el criterio 3 para disociación clásica mientras que el paciente 14 lo hizo para disociación fuerte. La discrepancia observada entre la repetición de prosodia emocional y lingüística en los pacientes 1, 4 y 6, en comparación con la discrepancia observada en los controles en las mismas, no presentó diferencias estadísticamente significativas por lo que no se cumplió el criterio 3 para ningún tipo de disociación. 
Tabla 3

Resultados del análisis con RSDT de la comparación de las discrepancias entre el rendimiento del paciente en las pruebas de comprensión de prosodia lingüística y emocional por un lado, y las pruebas de repetición de prosodia lingüística y emocional por el otro, y la discrepancia observada en el grupo control entre las mismas tareas (criterio 3: disociación clásica o fuerte)

\begin{tabular}{|c|c|c|c|c|c|c|c|c|}
\hline \multirow[b]{2}{*}{ Paciente } & \multicolumn{2}{|c|}{$\begin{array}{l}\text { Rendimiento } \\
\text { significativamente menor } \\
\text { que los controles (SI/NO) }\end{array}$} & \multicolumn{2}{|c|}{$\mathrm{RSDT}^{\mathrm{a}}$} & \multicolumn{2}{|c|}{$\begin{array}{c}\text { Rendimiento } \\
\text { significativamente menor } \\
\text { que los controles (SI/NO) }\end{array}$} & \multicolumn{2}{|c|}{$\mathrm{RSDT}^{\mathrm{a}}$} \\
\hline & $\begin{array}{l}\text { Lingüística } \\
\text { comprensión }\end{array}$ & $\begin{array}{l}\text { Emocional } \\
\text { comprensión }\end{array}$ & $t$ & $p$ & $\begin{array}{c}\text { Lingüística } \\
\text { repetición }\end{array}$ & $\begin{array}{l}\text { Emocional } \\
\text { repetición }\end{array}$ & $t$ & $p$ \\
\hline $1 \mathrm{MA}$ & SÍ & SÍ & 1.829 & .089 & SÍ & NO & 1.912 & .077 \\
\hline $2 \mathrm{ML}$ & SÍ & SÍ & .292 & .775 & NO & NO & 1.413 & .180 \\
\hline 3GL & NO & SÍ & 5.730 & $<.001 * * *$ & NO & SÍ & 3.493 & $.004 * *$ \\
\hline $4 \mathrm{MQ}$ & NO & NO & 1.115 & .283 & SÍ & NO & 2.097 & .055 \\
\hline $50 P$ & NO & SÍ & 1.388 & .187 & NO & SÍ & 11.562 & $<.001 * * *$ \\
\hline $6 \mathrm{MM}$ & NO & NO & .223 & .827 & NO & SÍ & 1.436 & .173 \\
\hline $7 \mathrm{JB}$ & SÍ & SÍ & 3.403 & $.004 * *$ & NO & SÍ & 11.901 & $<.001 * * *$ \\
\hline 8JP & NO & NO & 1.737 & .104 & NO & NO & .075 & .941 \\
\hline 9MR & NO & NO & .392 & .701 & NO & SÍ & 4.030 & $.001 * *$ \\
\hline 10GA & NO & NO & .278 & .785 & NO & NO & .000 & 1.000 \\
\hline $11 S J$ & NO & NO & .050 & .960 & $\mathrm{NO}$ & SÍ & 3.100 & $.009 * *$ \\
\hline $12 \mathrm{BA}$ & SÍ & SÍ & 6.835 & $<.001 * * *$ & NO & SÍ & 8.905 & $<.001 * * *$ \\
\hline $13 \mathrm{AG}$ & NO & NO & .139 & .892 & NO & NO & .216 & .832 \\
\hline 14SQ & SÍ & NO & 1.114 & .284 & SÍ & SÍ & 3.415 & $.004 * *$ \\
\hline 15NJC & NO & NO & 1.157 & .267 & SÍ & NO & 5.422 & $<.001 * * *$ \\
\hline 16CL & NO & NO & .412 & .687 & NO & NO & .105 & .918 \\
\hline 17GI & NO & NO & .157 & .877 & $\mathrm{NO}$ & NO & .000 & 1.000 \\
\hline
\end{tabular}

${ }^{a}$ RSDT: Revised Standarized Difference Test. Nivel de significación estadística: * $p<.05 ; * * p<.01 ; * * * p<.001$

\section{Resumen de disociaciones y patrones de disociaciones dobles.}

En la tabla 4 se muestra el resumen de cumplimientos de criterios de disociaciones clásicas y fuertes entre prosodia lingüística y emocional en las pruebas de comprensión para cada uno de los pacientes evaluados. Como se puede observar en dicha tabla, la mayoría de los pacientes no cumplió la totalidad de los criterios para determinar disociaciones entre ambos tipos de prosodia respecto de la habilidad de comprensión. Además, no se encontró ningún patrón de disociación doble ya que hubo un único paciente (caso 3) con una disociación clásica que evidenció alteración de la capacidad de comprender prosodia emocional pero no de prosodia lingüística, y no se halló a ningún paciente con el patrón opuesto. 


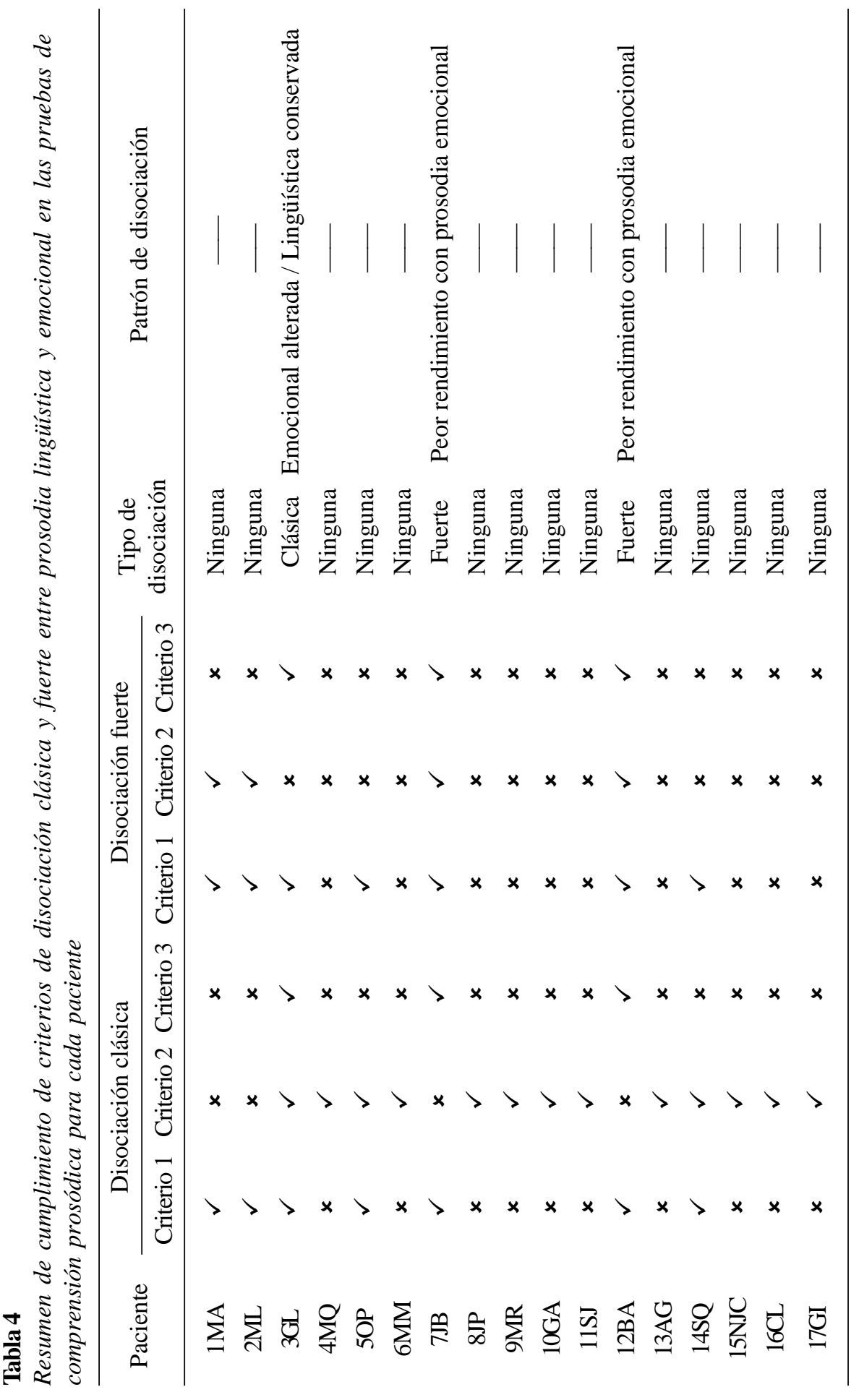


Respecto de la capacidad para repetir prosodia lingüística y emocional, en la tabla 5 se muestra el resumen de cumplimiento de criterios de disociación entre ellas para cada paciente. Como se puede observar, la mitad de los pacientes cumplió con los criterios para rendimiento disociado entre la capacidad de repetir ambos tipos de prosodia. En siete de los ocho pacientes en los que se observaron disociaciones, el patrón es el mismo: alteración para repetir prosodia emocional con conservación (o mejor rendimiento) de prosodia lingüística. Por otro lado, hubo un solo caso, el paciente 15 , en el que se observó el patrón opuesto (alteración en la repetición de prosodia lingüística con conservación de la repetición de prosodia emocional). La consideración conjunta de patrones de disociación de aquellos con rendimiento alterado para la prosodia emocional y conservado para la lingüística (e.g., paciente 3), en contraposición con el patrón inverso que presentó el paciente 15, evidencia la presencia de una disociación doble entre prosodia emocional y lingüística en la habilidad de repetición. 


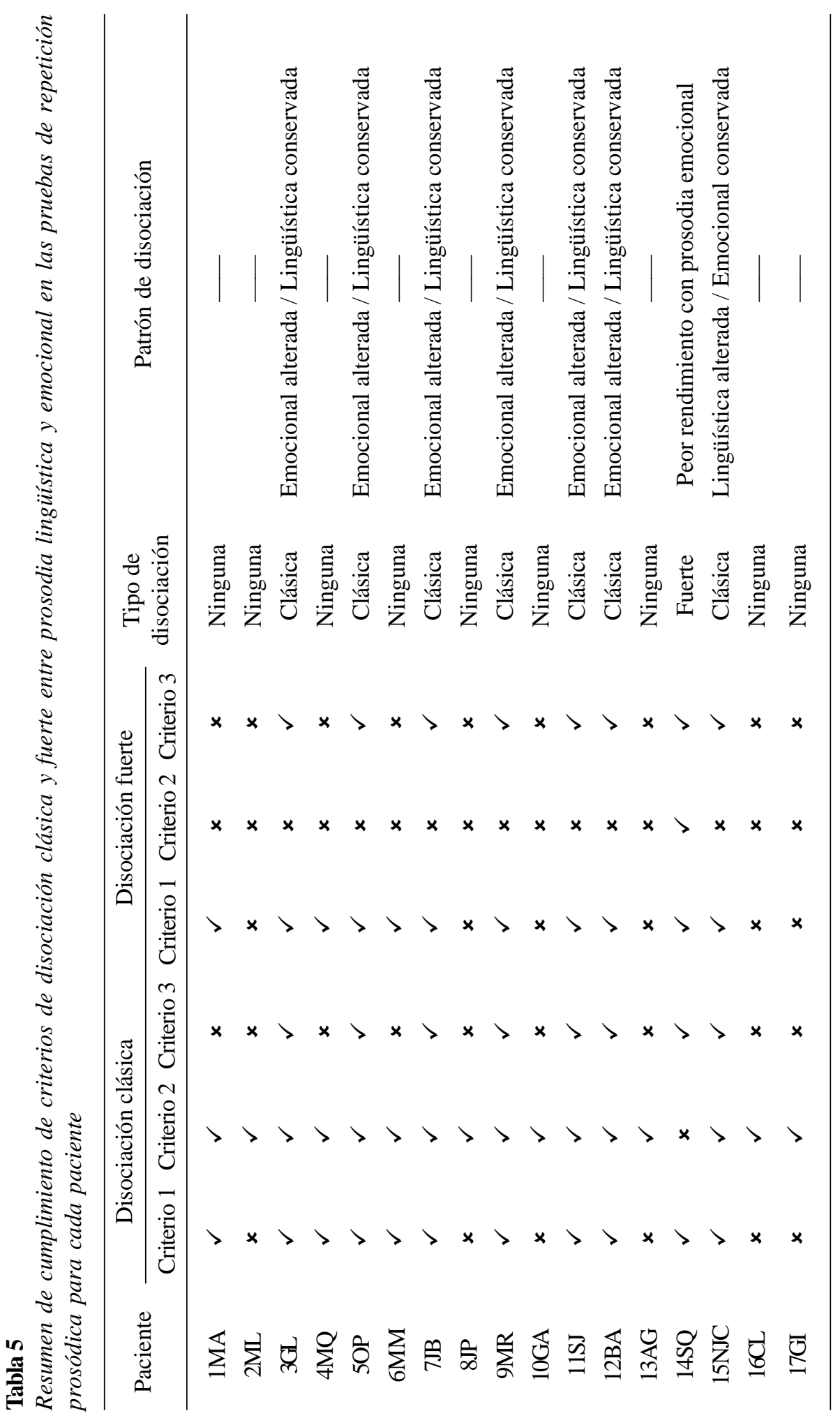




\section{Discusión}

El objetivo de este trabajo fue realizar un estudio de múltiples casos únicos para analizar patrones de disociaciones clásicas, dobles y fuertes entre el procesamiento de prosodia emocional y lingüística en pacientes con lesiones del HD. Los resultados obtenidos mostraron patrones de disociación fuerte y clásica entre ambos tipos de prosodia tanto en la habilidad de comprensión como de producción prosódica y, además, un patrón de doble disociación en producción prosódica.

Respecto de la habilidad para comprender, nuestros resultados mostraron que solo tres pacientes cumplieron criterios de disociación. En uno de ellos se observó disociación clásica entre prosodia emocional y lingüística, con afectación en el procesamiento de la primera y conservación de la segunda. En los otros dos pacientes, se observaron patrones de disociación fuerte con mayor afectación en la comprensión de la prosodia emocional que en la lingüística. Finalmente, no se halló ningún paciente con un patrón de rendimiento opuesto por lo que no se pudo establecer la existencia de una doble disociación emocional-lingüística en la comprensión de la prosodia en nuestro grupo de pacientes con lesiones del HD.

Investigaciones previas habían descripto fallas en los pacientes con lesiones del HD en la habilidad de comprensión de prosodia, tanto emocional (Dara, Bang, Gottesman, \& Hillis, 2014; Harciarek et al., 2006; Kho et al., 2008; Rymarczyk \& Grabowska, 2007; Wright et al., 2016) como lingüística (Parola et al., 2016; Pell, 1998), pero no habían examinado la existencia de diferentes patrones de rendimiento entre ellas. Estas evidencias permitieron documentar el compromiso de ambos tipos de comprensión prosódica en pacientes con lesiones del HD pero no fueron suficientes para fundamentar la existencia de independencia funcional entre prosodia emocional y lingüística. Los patrones de disociación hallados en el presente trabajo pueden considerarse evidencia a favor de la independencia funcional entre la capacidad de comprender prosodia lingüística y emocional, tal y como habían sugerido varios investigadores (Belyk \& Brown, 2014; Caballero, 2016; Joanette et al., 2008; Monrad, 1947; Ross \& Monnot, 2008; Witteman et al., 2014; Witteman et al., 2011; Wright et al., 2016). Sin embargo, al no hallarse en nuestro grupo de pacientes con lesiones del HD el patrón de disociación doble en la comprensión prosódica, queda pendiente la realización de trabajos futuros en los que se indague estos patrones en un número mayor de pacientes y/o en otras poblaciones patológicas para apoyar la hipótesis de la independencia funcional con evidencias de doble disociación.

Por otro lado, en las tareas de repetición de ambos tipos de prosodia, lo resultados mostraron que en siete pacientes se cumplieron criterios para disociaciones clásicas. En seis de ellos se observaron dificultades para repetir prosodia emocional mientras que la repetición de prosodia lingüística se encontraba conservada. Finalmente, se halló un paciente cuyo rendimiento mostró el patrón inverso (alteración de la repetición de la prosodia lingüística y conservación de la repetición de la prosodia emocional). El hallazgo de una disociación doble entre la capacidad de repetir prosodia emocional y lingüística apoya el supuesto de independencia funcional entre la repetición de ambas clases de prosodia ya que la alteración de un tipo de ellas no implica necesariamente la alteración en la otra y viceversa. En trabajos anteriores se reportó que los pacientes con lesiones de HD pueden presentar alteraciones tanto para producir prosodia emocional (Cohen et al., 1994; Guranski \& Podemski, 2015; Heilman et al., 2004; Wright et al., 2016) como lingüística (Ross, Shayya, \& Rousseau, 2013; Shah, Baum, \& Dwivedi, 2006). Por su parte, los resultados del presente trabajo permiten establecer, además, que esas alteraciones pueden mostrar una doble disociación que aportan evidencia a favor del postulado de independencia funcional entre ambos tipos de prosodia a nivel de la capacidad de producción. Los distintos patrones de alteración sugieren que existen procesos subyacentes diferenciados para la capacidad de producir prosodia 
emocional y lingüística y, por lo tanto, son pasibles de afectación independiente en pacientes con lesiones cerebrales. Estos datos son compatibles con aquellos trabajos realizados con imágenes funcionales que mostraron áreas cerebrales comunes pero también específicas para el procesamiento de prosodia emocional y lingüística (Belyk \& Brown, 2014).

Según el conocimiento de las autoras, los resultados reportados en el presente trabajo son los primeros en describir patrones de disociaciones clásicas, fuertes y dobles entre prosodia emocional y lingüística utilizando el diseño de casos-controles propuesto por Crawford y Garthwaite (2005). Y, si bien estos apoyan las presunciones teóricas clásicas de independencia funcional entre ambos tipos de prosodia, existen ciertas limitaciones que deberían ser tenidas en cuenta en la realización de trabajos futuros. En primer lugar, una de las limitaciones de este trabajo es que se incluyó un único grupo de pacientes (aquellos con lesiones del HD) por lo que sería importante ampliar la muestra hacia otros grupos patológicos, por ejemplo, a aquellos con lesiones del HI, con el fin de analizar patrones de disociación en dicho grupo. Como se puede observar en los resultados del presente trabajo, la mayoría de los patrones de disociaciones, excepto en un solo caso (paciente 15), mostró afectación de los aspectos emocionales y no de los lingüísticos. El sesgo de la composición muestral que incluye un grupo de pacientes con lesiones únicas del HD podría explicar este patrón tan marcado ya que se considera que este hemisferio sería dominante para el procesamiento emocional en general (Borod, Koff, \& Caron, 1983). Por otro lado, es sabido que el HI tiene un rol dominante para el procesamiento lingüístico en general. Algunos estudios recientes han ampliado las evidencias disponibles respecto de los déficits prosódicos en pacientes con lesiones del HI y sugieren que, en este grupo, también se observarían alteraciones de procesamiento prosódico (Leung, Purdy, Tippett, \& Leão, 2017; Mattavelli et al., 2017). Es por ello que la inclusión tanto de pacientes con lesiones del HD como del HI en trabajos futuros permitiría, quizás, observar patrones de alteración y disociaciones diferentes a los hallados en el presente trabajo.

Otras limitaciones de nuestro trabajo refieren a los tipos de estímulos utilizados ya que las pruebas de procesamiento prosódico del Protocolo MEC aquí utilizadas incluyen, únicamente, expresiones de tres emociones y estímulos prosódicos lingüísticos con variaciones en la modalidad. Respecto de la prosodia emocional, sería conveniente incluir otras emociones como miedo o sorpresa y analizar la posible presencia de patrones de disociaciones entre ellas. Por otro lado, respecto de la prosodia lingüística, se sugiere incluir en trabajos futuros estímulos que incluyan variedades de estímulos prosódicos con acento léxico y enfático. De esta forma, se podrá analizar si la independencia entre prosodia emocional y lingüística se sostiene al incluir otros aspectos prosódicos no evaluados en el presente trabajo.

Finalmente, en este artículo se describieron distintos patrones de disociación del procesamiento de la prosodia emocional y lingüística en un grupo de pacientes con lesiones del hemisferio derecho. Los resultados revelaron la existencia de patrones de disociaciones clásicas, dobles y fuertes, lo cual se convierte en evidencia a favor de la independencia funcional del procesamiento de ambos tipos de prosodia. 


\section{Referencias}

Becker, N., de Lima, J., de Carvalho Rodrigues, J., Villavicencio, A., \& de Salles, J. (2014). Estratégias de evocação lexical com critério semântico em adultos após acidente vascular cerebral no hemisfério direito. Letrônica, 7(1), 325-347. doi: 10.15448/1984-4301.2 014.1.16838

Belyk, M., \& Brown, S. (2014). Perception of affective and linguistic prosody: an ALE meta-analysis of neuroimaging studies. Social Cognitive and Affective Neuroscience, 9(9), 1395-1403. doi: 10.1093/scan/nst124

Borod, J. C., Koff, E., \& Caron, H. (1983). Right hemisphere specialization for the expression and appreciation of emotion: a focus on the face. In Perecman E., (ed). Cognitive processing in the right hemisphere (pp. 83110). New York: Academic Press.

Caballero, J. A. (2016). Organización cerebral de la prosodia. Estudios de Lingüística Aplicada, 63, 169196. doi: 10.22201/enallt.01852647p.2016.63.630

Casarin, F. S., Scherer, L. C., de Mattos, M. A., Ferré, P., ... Fonseca, R. P. (2014). Bateria Montreal de Avaliação da Comunicação-versão abreviada-Bateria MAC Breve. São Paulo: Pró-Fono.

Cohen, M. J., Riccio, C. A., \& Flannery, A. M. (1994). Expressive aprosodia following stroke to the right basal ganglia: a case report. Neuropsychology, 8(2), 242-245. doi: 10.1037/0894-4105.8.2.242

Crawford, J. R., \& Garthwaite, P. H. (2002). Investigation of the single case in neuropsychology: confidence limits on the abnormality of test scores and test score differences. Neuropsychology, 40(8), 1196-1208. doi: 10. 1016/s0028-3932(01)00224-X

Crawford, J. R., \& Garthwaite, P. H. (2005). Testing for Suspected Impairments and Dissociations in SingleCase Studies in Neuropsychology: Evaluation of Alternatives Using Monte Carlo Simulations and Revised Tests for Dissociations. Neuropsychology, 19(3), 318-331. doi: 10.1037/0894-4105.19.3.318

Crawford, J. R., \& Garthwaite, P. H. (2012). Single-case research in neuropsychology: A comparison of five forms of t-test for comparing a case to controls. Cortex, 48(8), 1009-1016. doi: 10.1016/j.cortex.2011.06.021
Crawford, J. R., Garthwaite, P. H., \& Porter, S. (2010). Point and interval estimates of effect sizes for the casecontrols design in neuropsychology: Rationale, methods, implementations, and proposed reporting standards. Cognitive Neuropsychology, 27, 245-260. doi: 10.1080/02643294.2010.513967

Crawford, J. R., \& Howell, D. C. (1998). Comparing an individual's test score against norms derived from small samples. The Clinical Neuropsychologist, 12, 482-486. doi: 10.1076/clin.12.4.482.7241

Dara, C., Bang, J., Gottesman, R. F., \& Hillis, A. E. (2014). Right hemisphere dysfunction is better predicted by emotional prosody impairments as compared to neglect. Journal of neurology \& translational neuroscience, 2(1), 1037.

de Lima, J., Becker, N., \& Fumagalli, J. (2014). Processamento léxico-semântico explícito e priming semântico em lesão cerebral de hemisfério direito. Ciências \& Cognição, 19(2), 119-139.

de Oliveira, C. R., Carlesso, K., de Feritas, L., Saloum, A., \& Fonseca, R. P. (2016). Processamento comunicativo em adultos com AVC unilateral de hemisfério direito: avaliação através de bateria breve. Acta Colombiana de Psicología, 19(2), 63-72. doi: 10.14718/ACP.20 16. 19.2.4

de Souza, M., Carlesso, K., Schwengber, F., Mineiro, A., ... Fonseca, R. P. (2015). Adaptation of the Montreal Communication Evaluation Battery to European Portuguese. Audiology-Communication Research, 20(3), 274-284. doi: 10.1590/S2317-64312015000200001563

Ellis, A. W., \& Young, A. W. (1992). Neuropsicología Cognitiva Humana. Barcelona: Masson S. A.

Fellows, A. L. (2013). Lesion Studies in Affective Neuroscience. En J. Armony, P. Vuilleumier (Eds), The Cambridge Handbook of Human Affective Neuroscience (p. 154-167). New York: Cambridge University Press.

Ferré, P., Clermont, M. F., Lajoie, C., Côté, H., Ferreres, A., Abusamra, V., ... Joanette, Y. (2009). Perfis clínicos de déficits comunicativos após acidente vascular cerebral de hemisfério direito: clusters transculturais. Neuropsicologia Latinoamericana, 1(1), 32-40. 
Ferré, P., Lamelin, F., Côté, H., Ska, B., \& Joanette, Y. (2011). Protocole MEC-P (Protocole Montréal d'Évaluation de la Communication (version de Poche). Isbergues: Ortho Édition.

Ferreres, A., Abusamra, V., Cuitiño, M., Côté, H., Ska, B., \& Joanette, Y. (2007). Protocolo MEC. Protocolo para la Evaluación de la Comunicación de Montreal. Buenos Aires: Neuropsi Ediciones.

Fonseca, R. P., Joanette, Y., Côté, H., Ska, B., Giroux, F., ... de Mattos, M. A. (2008a). Brazilian version of the Protocole Montréal d'Evaluation de la Communication (Protocole MEC): normative and reliability data. The Spanish journal of psychology, 11(2), 678-688.

Fonseca, R. P., Parente, M. A. M. P., Côté, H., Ska, B., Joanette, Y., \& Ferreira, G. D. (2008b). Bateria Montreal de Avaliação da Comunicação - Bateria MAC. Barueri. Brasil: Pró-Fono.

Guranski, K., \& Podemski, R. (2015). Emotional prosody expression in acoustic analysis in patients with right hemisphere ischemic stroke. Neurologia $i$ Neurochirurgia Polska, 49(2), 113-120. doi: 10.1016/ j.pjnns.2015.03.004

Harciarek, M., Heilman, K. M., \& Jodzio, K. (2006). Defective comprehension of emotional faces and prosody as a result of right hemisphere stroke: Modality versus emotion-type specificity. Journal of the International Neuropsychological Society, 12, 774-781. doi: 10.10170S1355617706060954

Heilman, K. M., Leon, S. A., \& Rosenbek, J. C. (2004). Affective aprosodia from a medial frontal stroke. Brain and Language, 89(3), 411-416. doi: 10.1016/j.bandl. 2004.01.006

Joanette, Y., Ansaldo, A. I., Kahlaoui, K., Côté, H., Abusamra, V., Ferreres, A., \& Roch-Lecours, A. (2008). Impacto de las lesiones del hemisferio derecho sobre las habilidades lingüísticas: perspectivas teórica y clínica. Revista de neurología, 46(8), 481-488.

Joanette, Y., Ska, B., \& Côté, H. (2004). Protocole Montréal d'évaluation de la communication (MEC). Isbergues, France: Ortho-Edition.
Kho, K. H., Indefrey, P., Hagoort, P., Van Veelen, C. W. M., van Rijen, P. C., \& Ramsey, N. F. (2008). Unimpaired sentence comprehension after anterior temporal cortex resection. Neuropsychologia, 46(4), 1170-1178. doi: 10.1016/j.neuropsychologia.2007.10.014

Lajoie, C., Ferré, P., \& Ska, B. (2010). O impacto do tipo de lesão nas alterações comunicativas pós-AVC de hemisfério direito. Neuropsicologia Latinoamericana, 2(3), 12-20.

Leung, J. H., Purdy, S. C., Tippett, L. J., \& Leão, S. H. (2017). Affective speech prosody perception and production in stroke patients with left-hemispheric damage and healthy controls. Brain and language, 166, 19-28. doi: 10.1016/j.bandl.2016.12.001

Mattavelli, G., Pisoni, A., Casarotti, A., Comi, A., Sera, G., Riva, M., ... Papagno, C. (2017). Consequences of brain tumour resection on emotion recognition. Journal of Neuropsychology. doi: 10.1111/jnp.12130

McIntosh, R. D., \& Brooks, J. L. (2011). Current tests and trends in single-case neuropsychology. Cortex, 47(10), 1151-1159. doi: 10.1016/j.cortex.2011.08.005

Monrand, G. H. (1947). Dysprosody or altered «melody of language». Brain, 70, 405-415.

Parola, A., Gabbatore, I., Bosco, F. M., Bara, B. G., Cossa, F. M., Gindri, P., \& Sacco, K. (2016). Assessment of pragmatic impairment in right hemisphere damage. Journal of Neurolinguistics, 39, 10-25. doi: 10.1016/ j.jneuroling.2015.12.003

Pell, M. D. (1998). Recognition of prosody following unilateral brain lesion: influence of functional and structural attributes of prosodic contours. Neuropsychologia, 36(8), 701-715. doi: 10.1016/S00283932(98)00008-6

Ross, E. D., \& Mesulam, M. M. (1979). Dominant Language Functions of the Right Hemisphere?. Prosody and Emotional Gesturing. Archives of neurology, 36(3), 144-148. doi: 10.1001/archneur. 1979.00500390062006

Ross, E. D., Shayya, L. \& Rousseau, J. F. (2013). Prosodic stress: Acoustic, aphasic, aprosodic and neuroanatomic interactions. Journal of Neurolinguistics, 26(5), 526-551. doi: 10.1016/j.jneuroling.2013.02.003 
Ross, E. D., \& Monnot, M. (2008). Neurology of affective prosody and its functional-anatomic organization in right hemisphere. Brain and Language, 104(1), 51-74. doi: 10.1016/j.bandl.2007.04.007

Rymarczyk, K., \& Grabowska, A. (2007). Sex differences in brain control of prosody. Neuropsychologia, 45(5), 921-930. doi: 10.1016/j.neuropsychologia.2006.08.021

Shah, A. P., Baum, S. R., \& Dwivedi, V. D. (2006). Neural substrates of linguistic prosody: evidence from syntactic disambiguation in the productions of braindamaged patients. Brain and Language, 96, 78-89. doi: 10.1016/j.bandl.2005.04.005

Shallice, T. (1988). From neuropsychology to mental structure. New York: Cambridge University Press.

Snow, D. (2000). The Emotional Basis of Linguistic and Nonlinguistic Intonation: Implications for Hemispheric Specialization. Developmental Neuropsychology, 17(1), 1-28. doi: 10.1207/S15326942DN1701_01

Witteman, J., Goerlich-Dobre, K. S., Martens, S., Aleman, A., Van Heuven, V. J., \& Schiller, N. O. (2014). The nature of hemispheric specialization for prosody perception. Cognitive, Affective, \& Behavioral Neuroscience, 14(3), 1104-1114. doi: 10.3758/s13415014-0255-1
Witteman, J., van IJzendoorn, M. H., van de Velde, D., van Heuven, V. J., \& Schiller, N. O. (2011). The nature of hemispheric specialization for linguistic and emotional prosodic perception: a meta-analysis of the lesion literature. Neuropsychologia, 49(13), 37223738. doi: 10.1016/j.neuropsychologia.2011.09.028

Wright, A. E., Davis, C., Gomez, Y., Posner, J., Rorden, C., Hillis, A. E., \& Tippett, D. C. (2016). Acute Ischemic Lesions Associated With Impairments in Expression and Recognition of Affective Prosody. Perspectives of the ASHA Special Interest Groups, 1(2), 82-95. doi: 10. 1044/persp1.SIG2.82

Zaidel, E., Kasher, A., Soroker, N., \& Batori, G. (2002). Effects of right and left hemisphere damage on performance of the «Right Hemisphere Communication Battery». Brain and language, 80(3), 510-535. doi: 10. 1006/brln.2001.2612

Zimmermann, N., Gindri, G., de Oliveira, C. R., \& Fonseca, R. P. (2011). Pragmatic and executive functions in traumatic brain injury and right brain damage: An exploratory comparative study. Dementia \& Neuropsychologia, 5(4), 337-345. doi: 10.1590/S198057642011DN05040013

\section{Samanta Leiva}

Facultad de Psicología, Universidad de Buenos Aires.

Doctoranda de la Facultad de Psicología de la Universidad de Buenos Aires. Becaria de doctorado UBACyT. Licenciada en psicología y especialista en neuropsicología clínica de la Universidad de Buenos Aires.

Autora corresponsal: leivasamanta@psi.uba.ar

\section{Micaela Difalcis}

Consejo Nacional de Investigaciones Científicas y Técnicas (CONICET).

Facultad de Psicología, Universidad de Buenos Aires.

Doctoranda de la Facultad de Filosofía y Letras de la Universidad de Buenos Aires. Becaria de doctorado CONICET. Profesora y licenciada en Letras de la Universidad de Buenos Aires.

micaeladifalcis@gmail.com

Cynthia López

Facultad de Psicología, Universidad de Buenos Aires.

Licenciada en psicología y especialista en neuropsicología clínica de la Facultad de Psicología, Universidad de Buenos Aires. Jefa de trabajos prácticos de la materia Neurofisiología, Facultad de Psicología, Universidad de Buenos Aires.

clopez@psi.uba.ar 


\section{Laura Margulis}

Facultad de Psicología, Universidad de Buenos Aires.

Unidad de Neuropsicología, Hospital Interzonal General de Agudos «Eva Perón».

Licenciada en psicología y especialista en neuropsicología clínica de la Facultad de Psicología, Universidad de Buenos Aires. Profesora adjunta de Neurofisiología, Facultad de Psicología, Universidad de Buenos Aires. Neuropsicóloga de la Unidad de Neuropsicología del Hospital Interzonal General de Agudos Eva Perón de Buenos Aires, Argentina.

lmargulis@psi.uba.ar

Andrea Micciulli

Unidad de Neuropsicología, Hospital Interzonal General de Agudos «Eva Perón».

Médica de la Facultad de Medicina de la Universidad de Buenos Aires. Especialista en Neurología y en Neuropsiquiatría. Médica Neuróloga de la Unidad de Neuropsicología del Hospital Interzonal General de Agudos Eva Perón de Buenos Aires, Argentina.

afmichu@hotmail.com

\section{Valeria Abusamra}

Consejo Nacional de Investigaciones Científicas y Técnicas (CONICET).

Facultad de Psicología, Universidad de Buenos Aires.

Facultad de Filosofía y Letras, Universidad de Buenos Aires.

Doctora en Letras por la Facultad de Filosofía y Letras de la Universidad de Buenos Aires. Investigadora Adjunta de CONICET. Profesora adjunta de la materia Psicolingüística de la Facultad de Filosofía y Letras, Universidad de Buenos Aires.

vabusamra@gmail.com

\section{Aldo Ferreres}

Facultad de Psicología, Universidad de Buenos Aires.

Unidad de Neuropsicología, Hospital Interzonal General de Agudos «Eva Perón».

Doctor en Psicología y médico de la Universidad de Buenos Aires. Jefe de la Unidad de Neuropsicología del Hospital Interzonal General de Agudos Eva Perón, Buenos Aires, Argentina. Director de la carrera de Especialización de Neuropsicología Clínica de la Universidad de Buenos Aires y Profesor Titular Regular de Neurofisiología de la Facultad de Psicología (UBA).

aferrere@psi.uba.ar

Declaración de conflictos de interés:

Los autores declaran no tener conflictos de interés asociados al presente trabajo. 